\title{
Exploring the High-altitude Nuclear Detonation and Magnetic Storms
}

\section{Mahmoud E Yousif*}

Department of Physics, The University of Nairobi, Nairobi, Kenya

\begin{abstract}
The High-altitude Nuclear Detonation (HND) experiments were carried between 1958/62 by both the United States of America (USA) and the former Union of Soviet Socialist Republics (USSR); the Starfish HND resulted in many phenomena, it discharged great amount of beta particles and debris, releasing intense gamma radiation which ionized atoms and molecules, resulting in Compton electrons and the production of Electromagnetic Pulse (EMP); the experiment also produced intense magnetic field, measured worldwide, with magnitude inversely proportional to square of the radial distance; the graph near the detonation center gave similar characteristics to the Magnetic Storms (MS); these characteristics were analyzed, whereas in both phenomenon, an External Magnetic Field-Moment $\left(E x M F_{M}\right)$ was produced; both the source and magnitude of the produced $E x M F_{M}$ were also analyzed and a mechanisms suggested for EMP production; characteristics regulating both phenomena are disclosed and elaborated from different perspectives; it is with hope that this will help in the process towards better understanding to some aspects related to the ExMF production in which if managed will be an advancement of the long awaited alternative renewable energy.
\end{abstract}

Keywords: High-altitude nuclear detonation; Magnetic fieldmoment production; External magnetic field; Magnetic storm; Interplanetary-external magnetic field; Electromagnetic pulse

\section{Introduction}

High-altitude Nuclear Detonation (HND) is a nuclear warhead detonated hundreds of kilometers above Earth's surface [1], it was carried by the United States of America (USA) and the former Union of Soviet Socialist Republics (USSR) between 1958-62. It produced complex phenomena such as magnetic disturbances, aurora and electromagnetic pulse (EMP) [2]. Before it was banned in 1962, the USA carried 13 tests [3], and 7 by USSR [4], the Starfish Prime experiment detonated at an altitude of $400 \mathrm{~km}$ South-South-West (SSW) of Johnston Island (JI) at 23:00 hours, Honolulu time on July 8, 1962; intended among others to disrupt an enemy of the anti-missile radars [2], with a total of thirteen experiments carried by USA at altitudes ranged from 11 to 540 kilometers [3], the nuclear detonation created a great amount of beta particles, debris and gamma rays which ionized more electrons [2], the Starfish Prime total Injected electrons were $1.3 \times 10^{25}$ electrons [4], yield 1.4 megatons $(\mathrm{mt})$ it caused minor damages of 300 street lights at Hawaii 1,445 km away, a probable failure of a microwave repeating station on Kauai, failure of the input stages of ionospheric sounders and damages to rectifiers in communication receivers [3], while the USSR Test 184, Operation K-3, on 22 October 1962, yield 300 kiloton (kt) burst at $290 \mathrm{~km}$ altitude near Dzhezkazgan, induced a current of 2,500 amps, this started a fire that burned down the Karaganda power plant, and shut down $1,000 \mathrm{~km}$ of shallow-buried power cables between Aqmola and Almaty [5], it injected 3.6x1025 electrons [4], producing intense electromagnetic pulse (EMP) and magnetic disturbances recorded in magnetic observatories worldwide [6], the K-3 damage was due to higher altitude and greater geomagnetic field [7], while Starfish Prim injected $7.5 \times 10^{25}$ electrons, $10 \%$ initially trapped in the radiation belts, while the rest were captured by the atmosphere [1], the Starfish Prim produced an unusual events in the great variety and extent of the phenomena [8], it produced maximum magnetic field of 700 gamma intensity after 70 seconds, although the physical processes caused the disturbances was ambiguously interpreted, but it was thought due to ionospheric currents attributed to the Magneto-hydrodynamic (MHD) [9], the magnetic disturbances was interpreted by others as resulted from an enhanced dynamo- action in the ionosphere, or a hydromagnetic waves resulted from geomagnetic field distortion [10], while some associated perturbations onset with MHD arrival caused by detonation debris containment the geomagnetic field, others interpreted geomagnetic field fluctuations and variations in E-layer ionization density as evidence that the perturbations were caused by nuclear radiations interactions with the magnetic field and ionosphere [3], records and analysis at a college in Alaska interpreted the strong perturbation at $66 \mathrm{sec}$ as an arrival of a transverse hydromagnetic wave generated in the magnetosphere in several earth radii [11].

Measurements of the Magnetohydrodynamic-Electromagnetic Pulse (MHD-EMP) from ionized fireball expansion to displace Earth's magnetic field in early stage of the test failed [1], due to the resulted speed and magnetic effect, hence it was concluded that the propagated disturbance is not explainable in terms of simple hydromagnetic waves, and the disturbance propagated electromagnetically in its most part [6], these magnetic disturbances didn't interfere with geophysical stations, and thought to improve understanding of the sudden commencements [3], where the magnetometer data at Johnston Island registered magnetic field of $150 \gamma, 270 \gamma$ and $-720 \gamma$ for Starfish Prim [9].

HND is investigated from spatial production of External Magnetic Field (ExMF) [12], relating HND with Magnetic Storms (MS) and the Disturbance Storm Time Index (Dst), fields magnitudes are derived, with suggested mechanism to produce them, the Storm Sudden Commencement (SSC) also analyzed, relating HND production with MS, and the Electromagnetic Pulse (EMP) [13] is also investigated suggesting a mechanism to generating it.

*Corresponding author: Mahmoud E Yousif, Department of Physics, The University of Nairobi, Nairobi, Kenya, Tel: +254 -715585050; E-mail: yousif_474@yahoo.com

Received June 03, 2014; Accepted July 02, 2014; Published July 16, 2014

Citation: Yousif ME (2014) Exploring the High-altitude Nuclear Detonation and Magnetic Storms. J Astrophys Aerospace Technol 2: 105. doi:10.4172/23296542.1000105

Copyright: $\odot 2014$ Yousif ME. This is an open-access article distributed under the terms of the Creative Commons Attribution License, which permits unrestricted use, distribution, and reproduction in any medium, provided the original author and source are credited. 
Citation: Yousif ME (2014) Exploring the High-altitude Nuclear Detonation and Magnetic Storms. J Astrophys Aerospace Technol 2: 105. doi:10.4172/2329-6542.1000105

Understanding both phenomenon will help in the efforts towards attaining the alternative renewable energy, which could reflect positively in environment and current imbalance in nature caused by excessive usage of fossil fuels.

\section{High-altitude Nuclear Detonations (HND) and Magnetic Storms (MS)}

The geomagnetic storms (GS) is defined as an "intense, irregular variability of geomagnetic field which occur as a consequence of solar disturbances," [14] but such variability in geomagnetic field had been produced through High-altitude Nuclear Detonation (HND), during which magnetic disturbances resulted from HND showed great resemblances with Magnetic Storms (MS) [1]; the GS affected electrical systems in a manner similar to the HND's third component (E3) generated by Electromagnetic Pulse (EMP) [15], both phenomena produced geomagnetic disturbances at magnetic observatories worldwide [6], they affected electrical and electronics systems [15]; thus with regard to time factor for each mechanism, the shape of both HND's magnetic field pattern recorded at Johnston Island shown in Figure 1 and Disturbance Storm Time Index (Dst) model [16], illustrates three common characteristics, these are: (1) The Storm Sudden Commencement (SSC), (2) main phase and (3) recovery phase; they differ in HND poses two sudden commencements, while the initial phase in HND is sharp, and magnetic disturbance due to HND sudden commencement would reach four times stronger than any recorded solar storms [1].

During MS Dst shown in Figure 2A, there are five SSC denoted by SSC- 1 to SSC-5, as measured at Honolulu, between 31 March and $3^{\text {rd }}$ April 1960; each SSC traced with dot line to Pioneer V at Figure 2B, where SSC-1, SSC-2 and SSC-3 were measured sometimes after Honolulu [17], while SSC-4 and SSC-5 delays were distorted by main

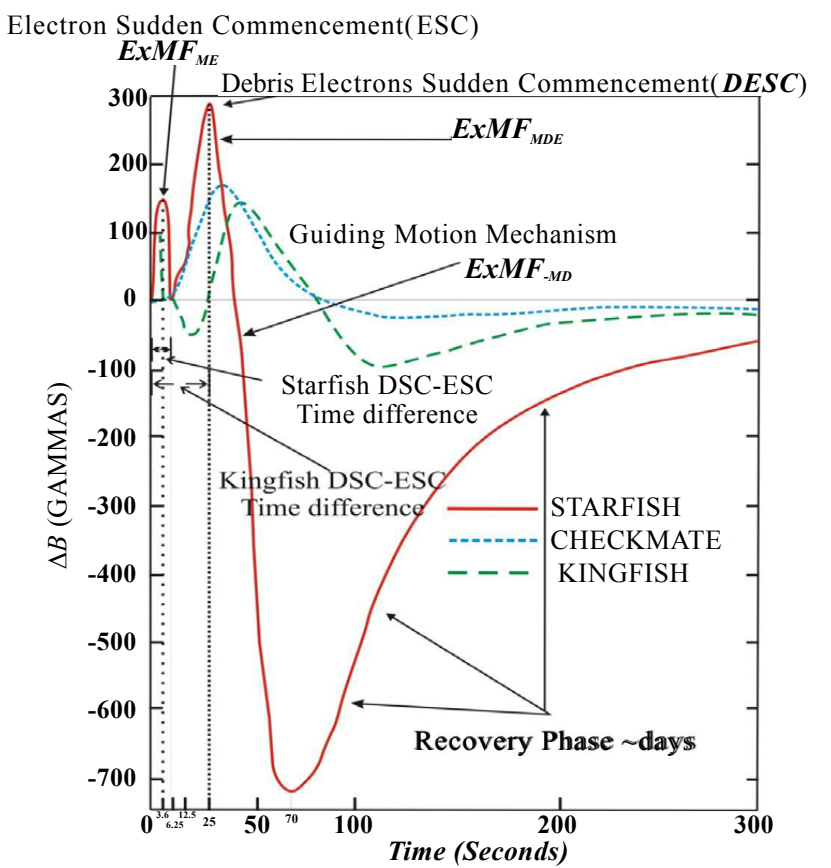

Figure 1: Graph of magnetic fields resulted from three High-altitude Nuclear Detonation (HND) measured at Johnston Island [1], HND's sequence of commencement and decay is similar to Storm Sudden Commencement (SSC) [16].

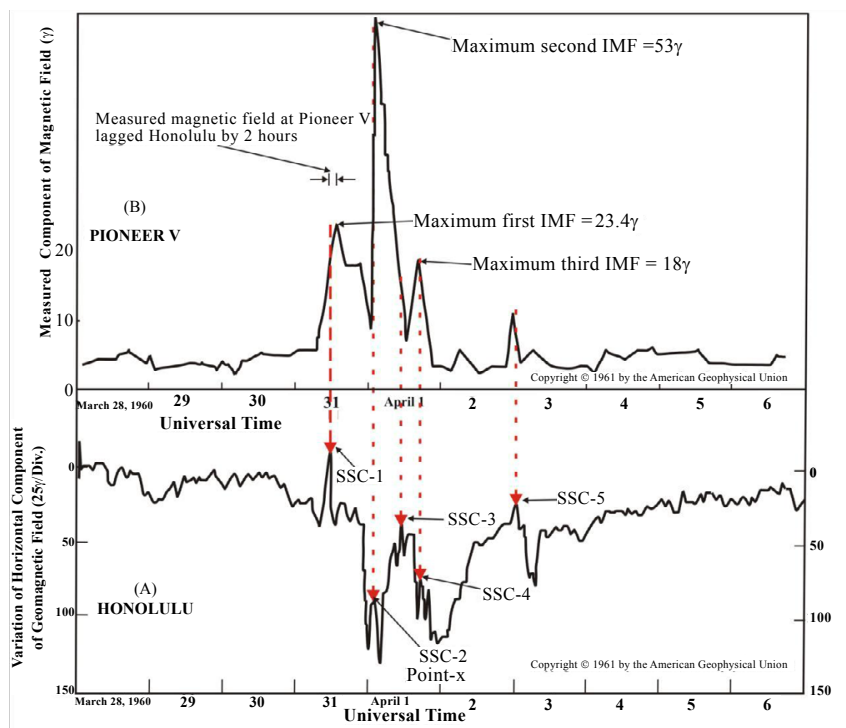

Figure 2: Each of Storm Sudden Commencement (SSC), measured at Honolulu during March/April 1960 Magnetic Storm (MS) shown in (A), also measured at Pioneer $V$ shown in (B) [17]. The Mechanism producing SSC relates to the High-altitudes Nuclear Detonation (HND) two Commencements in Figure 1; each Commencement is produced by charged particles clusters.

fields; thus each SSC followed by a drop in geomagnetic field, and beginning of the main phase, which lasted for a short period, due to re-occurrence of the new SSC; it is evidently that they intermingle due to successive SSC, resulting from successive solar wind clusters occurrence; this is similar to HND two sudden commencement shown in Figure 1, they are the designated Electrons Sudden Commencement (ESC) and Debris Electrons Sudden Commencement (DESC) respectively.

Since the concept of solar atmosphere extension and expansion into interplanetary space [18] endorsed by Pioneer V [19], showed to contain loopholes [20], and Magnetosphere breached continually by solar wind in several places [21], it is suggested that, both the flow of energetic protons [22], and energetic electrons $>200 \mathrm{keV}$ [23], in Magnetosheath, and the concentration of high density solar wind positive ion ranging between 35 to $127 \mathrm{~cm}^{-3}$ at Magnetosheath [22], contrary to background proton's density of $5-10 \mathrm{~cm}^{-3}$ [24], and Magnetosheath plasma interaction with magnetosphere at magnetopause, which results in fluctuations and strong magnetic fields, leading to an intense geomagnetic storm Dst $<200 \mathrm{n}$ Tesla [25], a situation which is similar to the ionospheric elongated diamagnetic cavity created after High-altitude Nuclear Detonation (HND) [1], therefore it is suggested that, both MS and HND are similar in nature, and resulted from charged particles interaction with geomagnetic field.

\section{The External Magnetic Field-Moment $\left(\operatorname{ExMF}_{M}\right)$}

The existence of magnetic lines of force and occurrence of magnetic disturbances are direct evidence related to the existence of a source of magnetic field; which was the origin of the Earth's dipole moment given by $\mathrm{M}=8.1 \times 10^{21}\left(\right.$ Tesla. $\left.^{3}\right)$ [26], while there are three estimates for magnetic moment of the ring current, Namely : $M=5.7 \times 10^{21}$ T.m ${ }^{3}$ (Smith and co-workers), $\mathrm{M}=1.8 \times 10^{21} \mathrm{~T} . \mathrm{m}^{3}$ (Model $\mathrm{V}_{2}$ belt), and $\mathrm{M}=4.1 \times 10^{20} \mathrm{~T} \cdot \mathrm{m}^{3}$ (Model $\mathrm{V}_{2}$ belt) [27].

The magnetic field strength resulted from Starfish Prim, measured at Hawaii, Samoa and Palo Alto shown in Figure 3, is inversely 


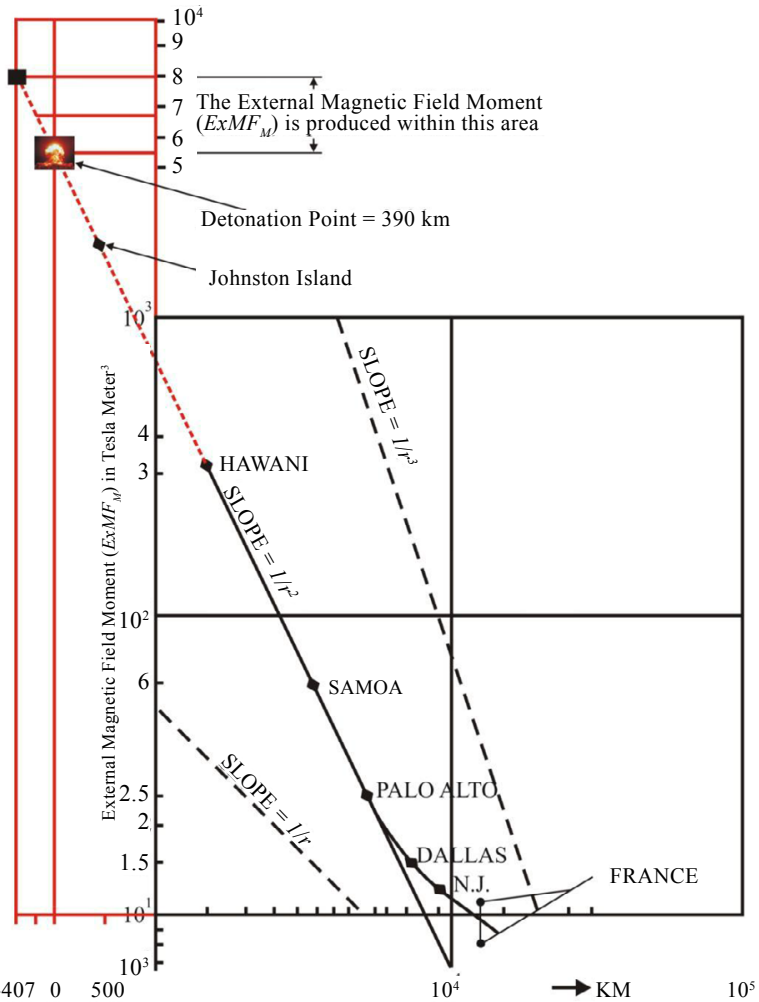

Figure 3: Magnetic field due to Starfish Prime, measured in Hawaii, Samoa and Palo Alto, it gives magnitude inversely proportional to square of the radial distance [1]. Tracing the slope $\left(1 / \mathrm{r}^{2}\right)$ to detonation point, gives the External Magnetic Field-Moment $\left(\right.$ ExMF $\left._{\mathrm{M}}\right)$.

\begin{tabular}{|c|c|c|c|c|c|c|}
\hline \multirow{2}{*}{$\begin{array}{l}\text { Event } \\
\text { Starfish }\end{array}$} & \multirow{3}{*}{\begin{tabular}{|l|} 
Distance \\
$1445 \mathrm{~km}$ \\
\end{tabular}} & \multicolumn{2}{|c|}{ HND and MS T } & \multirow{2}{*}{$\begin{array}{l}\text { ExMF }_{M} \text { in } \\
\text { T.M }{ }^{3} \text { Eq. } \\
\text { (1) } \\
313,251\end{array}$} & \multirow{2}{*}{$\begin{array}{c}\text { Number of } \\
\text { Charged } \\
\text { Particles } n_{m} \\
\text { Eq. }(5)\end{array}$} & \multirow{2}{*}{$\begin{array}{c}\begin{array}{c}\text { Storms vs } \\
\text { Starfish } \\
\text { Prim }\end{array} \\
1.4 \mathrm{MT}\end{array}$} \\
\hline & & \multirow{2}{*}{$\begin{array}{c}\text { ESC } \\
\text { Electrons }\end{array}$} & 150 & & & \\
\hline & & & 300 & 626,508 & $4.85 \times 10^{18}$ & \\
\hline \multirow{2}{*}{$\begin{array}{l}\text { Magnetic } \\
\text { Storm }\end{array}$} & \multirow[t]{2}{*}{$12.5 \mathrm{R}_{\mathrm{E}}$} & \multirow{2}{*}{$\begin{array}{c}\text { SSC } \\
\text { Protons }\end{array}$} & 159 & $1.0 \times 10^{9}$ & $7.9 \times 10^{33}$ & 1,596 MT \\
\hline & & & 214 & $1.4 \times 10^{9}$ & $1.1 \times 10^{34}$ & 2,234 MT \\
\hline
\end{tabular}

Table 1: Magnitudes of produced External Magnetic Field-Moment $\left(E \times M_{M}\right)$ around $400 \mathrm{~km}$ above $\mathrm{Jl}$, and the Interplanetary External Magnetic Field (I-ExMF) at 12.5 $R$ in Magnetosheath using Eq. (1), derived from SSC of $214 \mathrm{nT}$ at Trivandrum (TRD) Station, and $159 \mathrm{nT}$ from Annamalainagar (ANN) Station on 20-8-1991 [36] also given are electrons numbers $n_{m}$ in volume of magnetic lines of force for HND and protons number $n$ in volume of magnetic lines of force for MS using Eq. $\{5\}$, and comparison of these Moment with Starfish Prim test total yield=1.4MT, using $605,577 \mathrm{~T} . \mathrm{m}^{3}$, hence SSC $214 \gamma=2.2$ giga Tesla (GT), given $B_{\mathrm{g}}-\mathrm{HND}=2.5 \times 10^{-5} \mathrm{~T}$ $B_{g}-M S=2.5 \times 10^{-7} \gamma, v_{c}=2000 \mathrm{~km} \cdot \mathrm{s}^{-1}, \mathrm{I}-\mathrm{HND}=10 \mathrm{~km}$, and $\mathrm{I}-\mathrm{MS}=30 \mathrm{~km}$.

proportional to square of the radial distance, and the field magnitude at Hawaii measured $300 \gamma$ [1], but what was the magnitude of the source producing such field? Since the ring current is only describing the main phase of the Dst [27], then what is the source of the Storm Sudden Commencement (SSC)?

As HND and MS are so related and having many similarities, and $1 / r^{2}$ extension line showed in Figure 3 gives a progressive magnetic field increments towards an area where spatial External Magnetic Field $(E x M F)$ is produced [12], consequently this inversely law can determine the total magnitude of produced External Magnetic FieldMoment $\left(\operatorname{ExMF}_{M}\right)$, and also suggested to represents magnitudes of produced SSC, given by

$$
E x M F_{M}=B_{E x} r_{x}^{2} T . m^{2}
$$

where, $B_{E x}$ is the measured External Magnetic field (ExMF) in places such as Hawaii, Samoa and Palo Alto, or at Magnetic Observatories, $r_{\mathrm{x}}$ is the radial distance from the source of produced magnetic field to the measured point, and the $E x M F_{M}$ is the produced External Magnetic Field-Moment in Tesla in cubic meter $\left(\mathrm{T}_{\mathrm{m}} \mathrm{m}^{3}\right)$, Table 1 , gives such dipole moment for both Starfish Electrons Sudden Commencement (ESC) and two Storm Sudden Commencement (SSC).

\section{Beta particles capturing process}

When a HND, an initial flash light and X-rays were released, it immediately ionized atoms and molecules in the ionosphere over several hundred kilometers [10], gamma and X-rays released from the first stage, ionizing electrons from atoms and molecules during the first nanoseconds [1], forming Compton scattering, resulted in gamma rays energy of $1.2 \times 10^{13} \mathrm{~J}$ for every 1 -megaton bomb [13], while rocket measurement of beta particles experienced severe transient radiation effect, till $\mathrm{H}+0.002$ second [28], the ionization process is not limited below the fireball [1] but occurred in all directions, exceeding $900 \mathrm{~km}$ altitude, as measurement of Rocket P-4 (located 1025 kilometers along the field line from the burst) which detected an increase in gamma flux at $\mathrm{H}+455$ milliseconds, at concurrently with large flux of high-energy electrons [28], while Compton electrons resulted from gamma rays ionization, were measured during the first 20 milliseconds after $\mathrm{H}-0$ [28], and since large percentage of the detonation products is ionized and can therefore interact with the geomagnetic field [1], and since the magnetic lines of force through the burst point intersected the magnetic equator at $\mathrm{L} \sim 1.15$ which is an altitude of 900 kilometers [8], and Satellite measurements placed the center of the long lived artificial belt at an $\mathrm{L}$ value of 1.2, where debris and betas particles are injected at high L values [28], and since magnetic field mechanism was thought to slow down Starfish Prim debris [8], and Starfish Prim propagated disturbances couldn't be explained in terms of simple hydromagnetic waves, and could be electromagnetic in nature [6], therefore the interaction of ionized electrons with geomagnetic lines of force resulted in magnetic force, leading to bulk electrons gyrating around and along the geomagnetic lines of force, with orbital arrangements, not related to hydromagnetic; requiring different approach from current approach [29], and as electrons are captured by the geomagnetic lines of force [4], hence energetic electrons resembles solar wind, each electron from (HND)/ or proton from (MS) produced Circular Magnetic Field $(C M F)$ around itself and along its path as shown in Figure 4, hence a mechanism for capture process, resulted in magnetic force [30] given by

$$
F_{m}=B_{g} B_{e / p} r_{m}^{2} c \sin \theta N
$$

Where, $B_{g}$ is the geomagnetic field, $B_{e / p}$ is the $C M F$ produced by electrons or ions, $r$ is the magnetic radius of the CMF, $c$ is speed of light, $\theta$ is the angle between the two fields and the magnetic force $F_{m}$ is in Newton.

\section{Gyration radius}

Electrons/protons captured by geomagnetic lines of force given by Eq.(2), has initial large gyrating radius due to balance of forces $\left(B e v=m v^{2} / r\right)$, given by

$$
r_{m}=\frac{m_{e / p} v_{c}}{q B_{E x}} m
$$

Where, $\mathrm{v}_{\mathrm{c}}$ is electron/ion velocity and $r_{m}$ is the magnetic radius given in Eq.(2). 


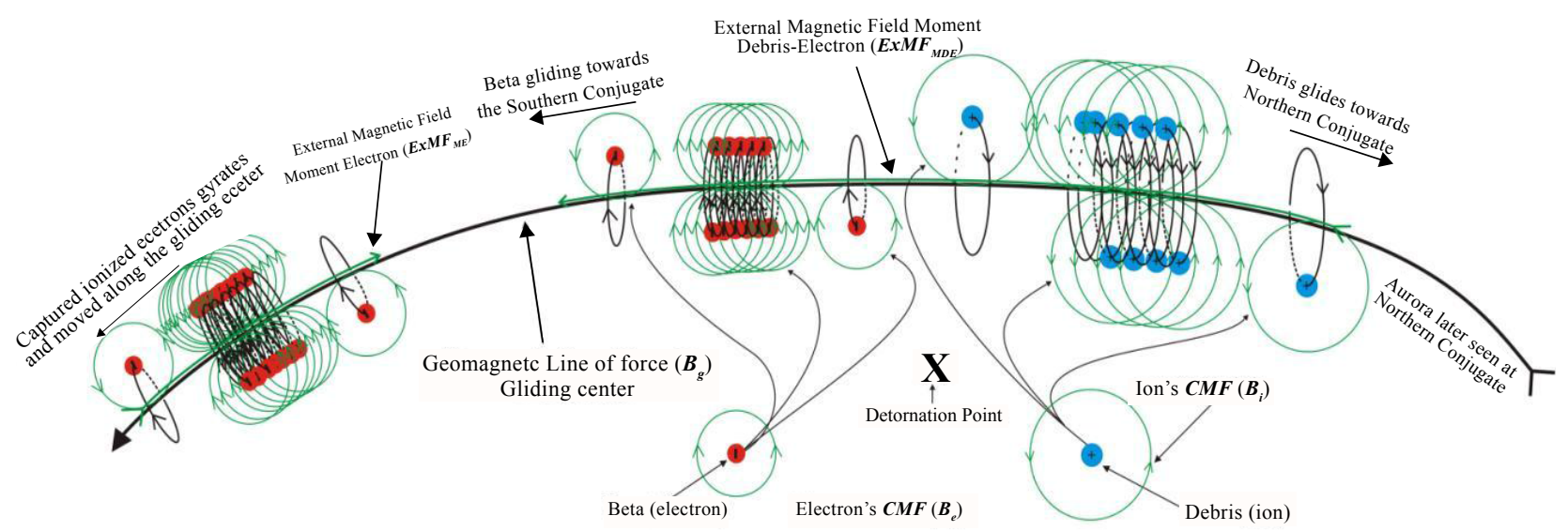

Figure 4: Beta and Debris particles gyrate around geomagnetic lines of force, producing an External Magnetic Field-Moment $\left(\right.$ ExMF $\left._{\mathrm{M}}\right)$ by the imposition of electron's and ion's Circular Magnetic Field (CMF) on the geomagnetic lines of force $\left(\mathbf{B}_{\mathrm{g}}\right)$ [30], after which particles glides oppositely towards related conjugate, producing aurora.

\section{External Magnetic Field Moment-Electrons $\left(\mathrm{ExMF}_{\mathrm{ME}}\right)$ Production}

An elongated diamagnetic cavity was produced within 1.2 seconds after a High-altitude Nuclear Detonation (HND). It expanded 1,840 $\mathrm{km}$ along the geomagnetic lines of force, having vertical high of 680 $\mathrm{km}$; and collapsed after 16 seconds [1], it was thought due to the dynamo effect resulting from strong air motions associated with the shock wave, and by a simultaneous enhanced conductivity in the lower ionosphere due to the detonation [10], linked with the appearance of beta auroras in the remote southern hemisphere conjugated, within a fraction of a second [31], where beta flux structure within the cavity was relatively uniform throughout and having $3 \times 10^{11}$ beta particles/ $\mathrm{cm}^{2} \mathrm{~s}$ for at least 7 seconds [1]; but an earlier Teak detonation, resulted in magnetic disturbances with vector axis at five central pacific stations directed approximately towards Johnston Island [10], it was interpreted as resulted from magnetic field lines become bunched up outside the fireball, due to the push-out [1], while the 700 gamma observed after 70 seconds shown in Figure 1, thought due to the upward motion (heave) of the atmosphere below the burst point [9], these loose ideas illustrate the existence of unknown astrophysical phenomenon, related to anomalous magnetic fields that is not well understood, the moment of which derived by Eq.(1), and required special treatments; differ from what is known.

On the other hand, three out of five magnetometers calibrated to $10^{4}$ gauss ( 1 Tesla) around the blast point, were affected by transient radiation [28], they failed to measure near the blast, while $400 \mathrm{~km}$ down at Johnston Island magnetic fields magnitudes of $150 \gamma, 290 \gamma$ and -720 $\gamma$ were measured [28], as shown in Figure 1, and measured worldwide, some of which are shown in Figure 3 [1]. As magnitudes of measured fields were inversely proportional to square of the radial distance; therefore by tracing the slop $1 / r^{2}$, from Palo Alto, to Samoa, and Hawaii, then to the spatial vicinity of the detonation, as traced by the extension red dot line showed in Figure 3, therefore great magnitudes are observed at/or around the blast point; hence the magnitude of the produced magnetic field near the source of production was much greater than the one Tesla (1Tesla) calibration, intended to measure small field changes, related to debris conductivity, and zero geomagnetic field inside the bubble, intended to understand debris-ambient interaction [32]; a miscalculation similar to Dr Hans A. Bethe's incorrect prediction, during the 1958 Teak and Orange nuclear blast, when instruments were set to measure longer and weaker Electromagnetic Pulse (EMP) with a different polarization [1], another similar situation occurred during a Starfish blast, when magnetometer on rocket Payload P-4 measured ambient magnetic field value of 0.22 gauss until $\mathrm{H}+0.68$ second, when the rubidium magnetometer electronic systems failed and thought due to transient radiation effects from high gamma flux [28], but the gamma radiation had already ionized electrons at that time, hence this resulted from rocket $\mathrm{P}-4$ presence near the source of produced External Magnetic Field-Moment $\left(E x M F_{M}\right)$, with magnitude given by Eq.(1), it was much intense than the less calibrated 1 Tesla magnetometer; such magnitude by $E x M F_{M}$ was only measured by Payload P-6, simultaneously with the arrival of the high-energy beta particles at $\mathrm{H} 1+51$ seconds which lasted for 12 mille-seconds [28]. A similar case to the above elongated diamagnetic cavity [1], was the concentration of high density solar wind positive ion ranging between 35 to $127 \mathrm{~cm}^{3}$ at Magnetosheath [22], contrary to background proton's density of 5-10 $\left(\mathrm{cm}^{3}\right)$ [24], that spatial point in Magnetosheath at $11.46 R_{E}$, is where magnetic field of $\sim 56 \gamma$ was measured by Interball [33]. Since the first large increase of magnetic field at Johnston Island was detected at time $\mathrm{H}-0$ [28], therefore electrons (for HND)/(protons for MS) captured and given by Eq.(2), instantly gyrate around the geomagnetic lines of force as shown in Figure 4, each producing Circular Magnetic Field $(C M F)$ or $B_{e}$, hence the addition of all $C M F$ produced HND's first initial phase, or the Electron Sudden Commencement (ESC) shown in Figure 1 and the initial phase for MS or the SSC, thus the instantly produced External Magnetic Field-Moment $\left(\operatorname{ExMF}_{M}\right)$ [34] given by

$$
E x M F_{M E / P}=10^{8}\left(B_{g x}^{2}+\frac{n_{m} l q^{3} B_{g}^{3}}{m_{e / p}^{2} v_{c} c}\right) \quad T
$$

Where, $10^{8}$ is the relative number of geomagnetic lines of force in square meter [35], $n_{m}$ is the number of gyrating electrons/protons in volume of geomagnetic lines of force, $l$ is the effective length of the magnetic lines of force around which electrons gyrates, $B_{g x}$ is the previous field intensity, $B_{g}$ is the geomagnetic field, $m_{e / p}$ electron or ion mass, $v_{c}$ is electron or proton velocity, and the produced External Magnetic Field-Moment Electron/Proton $\left(E x M F_{M E / P}\right)$, in Tesla.m ${ }^{3}$, as shown in Table 1, this equation gives the produced magnetic fields for both HND's Electron Sudden Commencement (ESC) shown in Figure 
Citation: Yousif ME (2014) Exploring the High-altitude Nuclear Detonation and Magnetic Storms. J Astrophys Aerospace Technol 2: 105. doi:10.4172/2329-6542.1000105

1, and Storm Sudden Commencement (SSC).

Substituting the right hand side of Eq.(1) with the left hand of Eq.(4), then rearranging, the number of electrons or ions $\left(n_{m}\right)$ in volume of magnetic lines of force producing the $E x M F_{M E / P}$ is given by

$$
n_{m}=\frac{B_{E x} r_{x}^{2} m_{e}^{2} v_{c} c}{10^{8} l q^{3} B_{g}^{3}}
$$

For small magnetic fields, where $B_{E x}$ equal to $E x M F_{M E}$, Eq.(5) becomes

$$
n_{m}=\left(\frac{B_{E x} m_{e}^{2} v_{c} c}{10^{8} l q^{3} B_{g}^{3}}\right)
$$

The magnetic field produced in unit of space, when electrons or protons are known is given by

$$
B_{E x}=\left(\frac{10^{8} n_{m} l q^{3} B_{g}^{3}}{m_{e}^{2} v_{c} c}\right) \quad T
$$

While distance from the detonation point, when electrons/protons numbers are known is given by

$$
r_{x}=\sqrt{10^{8}\left(\frac{n_{m} l q^{3} B_{g}^{3}}{B_{E x} m_{e}^{2} v_{c} c}\right)} \quad m^{2}
$$

\section{Electromagnetic Pulse (EMP) generation}

The nuclear Electromagnetic Pulse (EMP) is a time varying electromagnetic radiation which rapidly increases to a peak then slowly decays [37], as shown in Figure 5, it consists of a sharp transient signal with a strong frequency component in the neighborhood of $15 \mathrm{kHz}$ [1], it was first realized after the $1.2 \mathrm{kt}$ Jangle-Sugar test in 1951, where over 1000 Amperes current fused the bomb control cables of $0.8 \mathrm{~km}$ distance in the Nevada Test Site [1]. EMP was studied with great concern due to damages that can inflict on electric and electronics systems, which could reflects on different sectors such as electric power, telecommunication, banking and finance, transportation infrastructure, food infrastructure, emergency service, space system and government [15], realizing these significance following the ratification of the Limited Test Ban Treaty (LTBT) that ended excessive release of nuclear fallout into the planet's atmosphere [38], scientists heavily dependence on computer assimilation, but Longmire and Gilbert [39] urged on theoretical understanding to avoid wrong answer to the right problem.

New EMP hypothesizes appeared after Starfish Prime test in 1962 [31], interpreting EMP as resulted from the primary Compton electrons and currents; the variation of which accelerate charge and radiated EMP [1]; above $30 \mathrm{~km}$, it was thought as resulting from Compton electrons deflected by interaction with geomagnetic field in the deposition region of 20-40 km [3], while according to KarzasLatter high altitude EMP model theory, Compton electrons are produced in specific region of the atmosphere known as the absorption region, it then turned by geomagnetic field to produce the EMP [40]. It was thought that Compton electrons radiated EMP through other mechanisms [41], but which mechanism? It is clear that there isn't a satisfactory answer to the mechanism behind EMP; and to what is it from $E x M F_{M E}$ perspective?

The first large increase of magnetic field at Johnston Island was at the time $\mathrm{H}-0$, it was due to the EMP, with inaccurate amplitude [28]. This increase in magnetic field is very significant, then the maximum 150

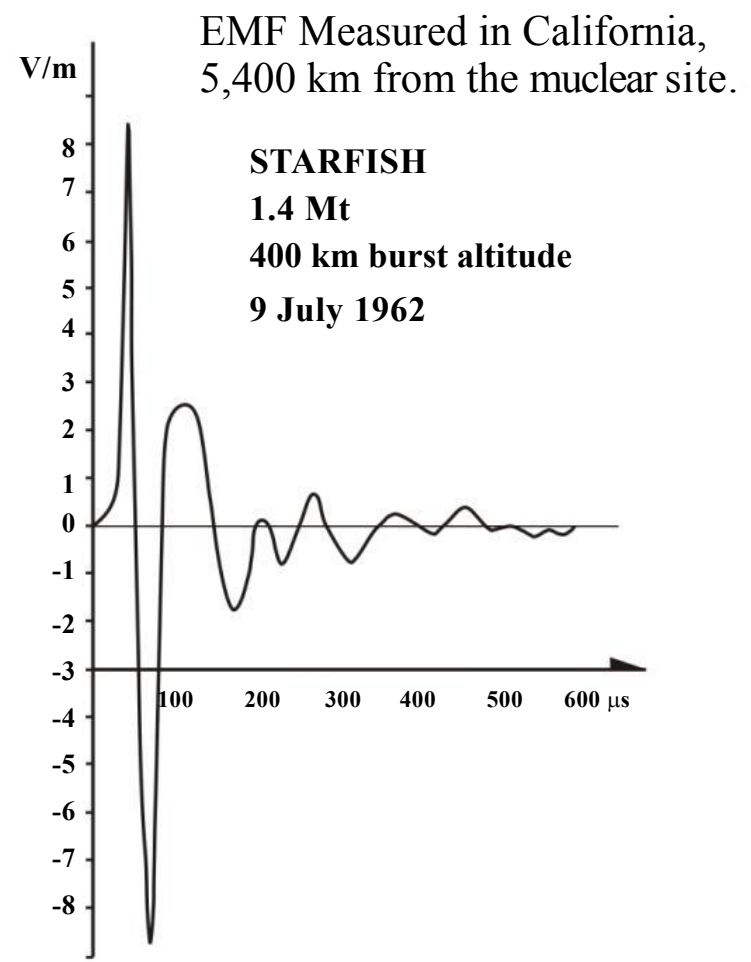

Figure 5: Starfish produced Electromagnetic Pulse (EMP), measured in California, 5,400 Km from the detonation point [1].

\begin{tabular}{|l|l|l|l|l|l|l|}
\hline Number $\left(\boldsymbol{n}_{\boldsymbol{m} 1}\right)$ & $3.6 \times 10^{8}$ & $3.6 \times 10^{9}$ & $3.6 \times 10^{10}$ & $3.6 \times 10^{11}$ & $3.6 \times 10^{12}$ & $3.6 \times 10^{13}$ \\
\hline
\end{tabular} \begin{tabular}{|l|l|l|l|l|l|l|l|}
\hline Produced $B_{E x} \mathbf{T}$ & $4.65 \times 10^{-5}$ & $4.65 \times 10^{-4}$ & $4.65 \times 10^{-3}$ & $4.65 \times 10^{-2}$ & $4.65 \times 10^{-1}$ & 4.65 \\
\hline
\end{tabular}

Table 2: Variation of produced magnetic field by variable electrons clusters.

$\gamma$ (from $3.425 \times 10^{-5} \mathrm{~T}$ to $3.44 \times 10^{-5} \mathrm{~T}$ ) which occurred at $\mathrm{H}+3.6$ seconds [28], this maximum was produced by Compton electrons, and it is the ESC, given by Eq.(4) and shown in Figure 1, but before attaining this $150 \gamma$ maximum, ESC $\left(B_{E x}\right)$ was progressively produced at an increment of: $\delta B_{E x 1}, \delta B_{E x 2}, \delta B_{E x 3}, \delta B_{E x 4}, . . \delta B_{E x n}$, by electrons clusters of: $n_{m 1}, n_{m 2}, n_{m 3}$, $n_{m 4}, \ldots n_{m n}$, these electrons didn't arrive all together at once, but arrived in sequential clusters, the first cluster produced the first large increase in magnetic field at time $\mathrm{H}-0$ related to EMP production [28], therefore which electrons number in the cluster produced the large increase?

Electrons number $\left(n_{m}\right)=3.6 \times 10^{18}$

electrons in volume of geomagnetic lines of force were suggested to contribute (coherently) to the EMP [41], using this in Eq.(7), given electron's velocity $v_{c}=2,000 \mathrm{~km}, l-\mathrm{HND}=10 \mathrm{~km}$, and geomagnetic field $B_{g}=2.5 \times 10^{-5} \mathrm{~T}$, the magnetic field magnitude obtained is $464,648.4$ T. $\mathrm{m}^{3}$; this magnitude is around what was given by Eq.(1), shown in Table 1, hence too big for the first cluster.

Since electrons number $\left(n_{m}\right)$ arrived in cluster, consists of millions of electrons, hence several possibilities should be tried, these are, given in Table 2: $n_{m 1}=3.6 \times 10^{8}, n_{m 1}=3.6 \times 10^{9}, n_{m 1}=3.6 \times 10^{10}, n_{m 1}=3.6 \times 10^{11}$, $n_{m 1}=3.6 \times 10^{12}$, and $n_{m 1}=3.6 \times 10^{13}$ electrons.

Substituting each of these possible clusters in Eq.(7), given electron's $v_{c}=2,000 \mathrm{~km}, l=10 \mathrm{~km}$ and $B_{g}=2.5 \times 10^{-5} \mathrm{~T}$, thus from Table 2, the ExMF or $B_{E x}=4.65 \times 10^{-3}$ Tesla is chosen.

Since the EMP strength increased with burst height and 
geomagnetic field [40], and at $1450 \mathrm{~km}$ away at Honolulu Starfish EMP gave $5,600 \mathrm{~V} \mathrm{~m}^{-1}$ at about 0.1 microseconds [1], and the intense EMP magnitudes occurred closer to the burst point [1] which could exceed $50 \mathrm{kV} \cdot \mathrm{m}^{-1}$ for Starfish [40], therefore the first electrons cluster thought to interact with geomagnetic field, given in Table 2, in yellow color, by $n_{m-1}=3.6 \times 10^{10}$ electrons in volume of geomagnetic lines of force, are the first Compton electrons, collectively interacted with the geomagnetic lines of force, producing the first large field at Johnston Island at time H-0 [28], with magnitude $B_{E x}=4.65 \times 10^{-3}$ Tesla, this instantly reduced gyrating radius given by Eq.(3), hence the new radius is

$$
r_{C 1}=\frac{m_{e} v_{c}}{q B_{E x}} \quad m
$$

Where, $r_{C 1}$ is the first Compton radius, and $B_{E x}$ is the produced $E x M F=4.65 \times 10^{-3}$ Tesla, it changed gyrating radius from 0.45 meter given by Eq. (2) to $2.45 \times 10^{-3} \mathrm{~m}(0.00274 \mathrm{~m})$ given by Eq.(9), thus this reduction accelerates electrons.

As the first electrons cluster of $n_{m-1}=3.6 \times 10^{10}$ electrons in volume of geomagnetic lines of force, produced strong $\delta B_{E x l}=4.65 \times 10^{-3} \mathrm{~T}$, within $\delta t_{1}=0.1 \mu \mathrm{s}$, hence this is expressed by

$$
E=\frac{\delta B_{E x 1}}{\delta t_{1}} V \cdot m^{-1}
$$

But such relation is the electrodynamics fields which generates electric field [42] and given by

$$
\nabla \times E=-\frac{\delta B}{\delta t} V \cdot m^{-1}
$$

However, since EMP is a time varying electromagnetic radiation [37], therefore these change produced an intense electromagnetic pulse (EMP) given by

$$
E M P 1=\nabla \times E=-\frac{\delta B_{E x 1}}{\delta t_{1}} V \cdot m^{-1}
$$

From above equation, $B_{E x}$ of 0.00245 Tesla, changed in 0.1 micro second, gives electric field intensity of $46,5 \mathrm{k} \mathrm{V} . \mathrm{m}^{-1}$.

Since the first cluster contain $n_{m-1}=3.6 \times 10^{10}$ electrons in volume of geomagnetic lines of force, this is only part of the whole given $3.6 \times 10^{18}$ electrons given by Longmire [41], but from Table 1, the total electrons number in the first ESC $n_{m}=2.43 \times 10^{18}$ electrons in volume of geomagnetic lines of force, therefore there are still $6.75 \times 10^{7}$ electrons to be interacted with the geomagnetic lines of force, these are divided in clusters; relating this with Figure 5, thus there are several less $\delta B_{E x n}$ ' $\delta t n$ EMP to be generated, therefore with each cluster generates EMP $\mathrm{EMP}_{2}, \mathrm{EMP}_{3}$ and $\mathrm{EMP}_{\mathrm{n}}$, therefore the total generated EMP is given by

$$
E M P=\nabla \times E=\left(\left(-\frac{\delta B_{E \times 1}}{\delta t_{1}}\right)+\left(-\frac{\delta B_{E \times 2}}{\delta t_{2}}\right)+\left(-\frac{\delta B_{E \times 3}}{\delta t_{3}}\right) \ldots . . \cdot\left(-\frac{\delta B_{E \times n}}{\delta t_{n}}\right)\right) V \cdot m^{-1}
$$

Substituting the right hand of Eq.(7) with $B_{E x}$ in Eq.(13) above, therefore EMP is given by

$$
E M P=\left(\frac{\delta\left(10^{8} n_{m} l q^{3} B_{g}^{3}\right)}{\delta\left(m_{e}^{2} v_{c} c t\right)}\right)+\left(\frac{\delta\left(10^{8} n_{m} l q^{3} B_{g}^{3}\right)}{\delta\left(m_{e}^{2} v_{c} c t\right)}\right)+\ldots . .\left(\frac{\delta\left(10^{8} n_{m} l q^{3} B_{g}^{3}\right)}{\delta\left(m_{e}^{2} v_{c} c t\right)}\right) V \cdot m^{-1}
$$

The frequency of the produced EMP is given by

$$
f_{\text {EMP }}=\frac{1}{t} \quad H z
$$

\section{External Magnetic Field-Moment Ions $\left(\mathrm{ExMF}_{\mathrm{MI}}\right)$ production}

HND earlier geomagnetic disturbances thought to have one initial phase [10], but as shown in Figure 1, Starfish Prim, Checkmate and Kingfish resulted magnetic field at Johnston Island, showed two initial phases, followed by a slow decrease and subsequent lengthy recovery to ambient conditions [28], the fields shown in Figure 1, are similar in shape to the Dst with one Sudden Storm Commencement (SSC) [16], the first initial phase of Starfish Prim shown in Figure 1, measured $150 \gamma$ at $\mathrm{H}+3.6$ seconds, followed by $290 \gamma$ at $\mathrm{H}+25$ [28]; comparing this mechanism, with energetic electrons $>200 \mathrm{keV}$ at Magnetosheath which related to the magnetopause electron layer [23], or Magnetosheath plasma, that interacts with the magnetosphere at the magnetopause, and fluctuates with strong magnetic field, leading to an intense geomagnetic storm with Dst $<200 \mathrm{n}$ Tesla [25], which is similar to the energetic Magnetosheath protons [22], or the energetic protons first detected by Pioneer V, and later caused geomagnetic disturbance measured at Honolulu and shown in Figure 2A [17], all of which suggesting the production of an Interplanetary External Magnetic Field $(I-E x M F)[12]$.

As ExMF $F_{M E}$ produced by Compton electrons given by Eq.(4), starts gliding along the guiding center towards southern conjugate, the fast upwards moving debris [2], which raised much faster to several hundred kilometers in Orange Detonation [3], with great intensity, rapidly expanding and arrived after beta particles [2]; these large positive ions flux have average velocity of $345 \mathrm{~km} / \mathrm{s}$ measured at $\mathrm{H}+1.20$ seconds, it continued for 400 seconds [28], where large fraction of these debris moved swiftly through field lines to $100 \mathrm{~km}$ above northern conjugate, other fraction moved to the southern conjugate area [3], and with sharp increase in beta flux intensity occurred at $\mathrm{H}+480$ milliseconds [28], the remained ionized ions travel outward more slowly [37], and most of the high-energy betas were injected along the field line [28], and with averaged beta particle radiation flux in the belt was $2 \times 10^{14}$ electrons per square meter per second, 3 minutes after burst [1], these demonstrates beta and ions mutual coexistence as shown in Figure 4.

When $E_{M M F_{M E}}$ given by Eq.(4), reached maximum magnitude of $150 \gamma$ [28], a time at which debris and beta particles start approaching to interact with geomagnetic lines of force, as shown in Figure 4, this showed there are two mechanisms producing the two initial phases of the HND, shown in Figure 1, these mechanisms are : (A) Electrons Sudden Commencement (ESC), given by Eq.(4), and (B) Debris/ Electrons Sudden Commencement (DESC); and since positive and negative charges particles were detected moving in opposite direction at $\mathrm{H}+6$ seconds [28], which is the time in Figure 1, at which the second DESC start building up, it is an indication that they oppositely gyrates along the geomagnetic lines of force as shown in Figure 4, hence producing the External Magnetic Field-Moment Debris Electrons $\left(E x M F_{M D E}\right)$, giving raise to the second initial phase or the DESC, with magnitude double that given by Eq.(4), it is given by

$$
E x M F_{M D E}=\left(10^{8}\left(B_{g x}^{2}+\frac{n_{m} l q^{3} B_{g}^{3}}{m_{e}^{2} v_{c} c}\right)+10^{8}\left(B_{g x}^{2}+\frac{n_{m} l q^{3} B_{g}^{3}}{m_{i}^{2} v_{c} c}\right)\right) T \cdot m^{2}
$$

Where, $m_{i}$ is ions mass, and the ExMF $F_{M D E}$ is the produced electrons and positive ions External Magnetic Field-Moment Debris Electrons $\left(E x M F_{M D E}\right)$ in T.m ${ }^{3}$, the produced $E x M F_{M D E}$ will not generates EMP, due to the existence of previous $E x M F_{M E}$ and gyrating radius is not reduced. The numbers of both electrons and ions in volume of magnetic lines of force generating $E x M F_{M E}$ is

$$
n_{m N}=\left(\frac{B_{E x} r_{x}^{2} v_{c} c}{10^{8} l q^{3} B_{g}^{3}}\right)\left(m_{e}^{2}+m_{i}^{2}\right) \quad T
$$


Citation: Yousif ME (2014) Exploring the High-altitude Nuclear Detonation and Magnetic Storms. J Astrophys Aerospace Technol 2: 105. doi:10.4172/2329-6542.1000105

\section{Gliding along geomagnetic lines of force (Guiding-Center)}

When Electrons Sudden Commencement (ESC) shown in Figure 1 , reached maximum magnitude, and since debris motion largely governed by the magnetic field [3], the produced high $B_{E x}$ magnitude attracted opposite gradients geomagnetic fields, hence electrons while gyrating, glides along geomagnetic lines of force (the guiding center) towards the southern conjugate as shown in Figure 4; at that moment the produced DESC, reached maximum ExMF $F_{M D E}$ given by Eq.(16), after which gyrating debris and beta particles glides along geomagnetic lines, towards the northern conjugate as shown in Figure 4, although some may glides towards the southern conjugate; the gliding force for both $E x M F_{M E}, E x M F_{M D E}$ and MS at $12.5 R_{E}$ is given by

$$
F_{G}=B_{g c} B_{E x} r_{m g}^{2} c \sin \theta \quad N
$$

Where, $B_{g c}$ is geomagnetic fields towards the conjugate, $r_{m g}$ is the radial distance at which the field is sensed, $\theta$ is the angle at which the field is sensed, and $F_{G}$ is the gliding force in Newton, Eq.(18) becomes

$$
m_{e / i} \frac{\delta v}{\delta t}=B_{g} B_{E x} r_{m g}^{2} c \sin \theta \quad N
$$

Since motion is in straight line, therefore the velocity along the guiding center is given by

$$
v=\frac{\left(B_{g} B_{E x} r_{m g}^{2} c \sin \theta\right) t}{\left(m_{e / i}\right)} m \cdot s^{-1}
$$

Instant aurora appeared in southern conjugate region after detonation [31], it first appeared between 4.1 and 6.7 millisecond [8], hence in connection with Figure 1, this showed how strong the produced force and related extremely fast velocity, because the gliding force was established during the building up of the ExMF $F_{M E}$; while for the Geomagnetic storm which is identified by the development of the ring current in the magnetosphere [14], though the origin of this current lack a satisfactory explanation [43], this is simply due to the strong force produced by Eq.(18), and the ions' exceptionally fast velocity while moving from Magnetosheath at $12.5 R_{E}$ to form ring current at around 5-7.5 $R_{E}$ [27], or around $\sim 3 R_{E}$ and $\sim 4-7 R_{E}$ for all levels of geomagnetic disturbances [44], it also shows how difficult to detect rig current formation, and many other ionosphere phenomena.

\section{The guiding center main phase magnetic moment}

While gliding along geomagnetic lines of force, $E x M F_{M E}$ produced a negative field, this is countered by newly produced positive DESC or the ExMF $F_{M D E}$, for Starfish the debris arrival period is short hence the negative produced field is superseded by $E_{X M F_{M D E}}$ as shown in Figure 1; but if the period for debris arrival is long such as in Kingfish as shown in Figure 1, the negatively produced guiding center appears for a while. The main negative External Magnetic Moment-Electrons Gliding Center $\left(\right.$ ExMF- $\left._{M E G}\right)$ (main phase for MS) is given by

$$
E x M F_{-M E G}=\left(B_{g}\right)-\left(10^{8}\left(\gamma_{P S} B_{g x}^{2}+\frac{\gamma_{P S} n_{m} l q^{3} B_{g}^{3}}{m_{e}^{2} v_{c} c}\right)\right) T
$$

Where, $\gamma_{\mathrm{PS}}$ is the relative magnitudes of both produced primary and secondary ExMF ( $P$ \& $S-E x M F$ ) it is obtained due to the small radius of gyration [34].

At that moment, ExMF $F_{-M D E G}$ starts opposing the geomagnetic field, while gliding along the northern geomagnetic lines of force for the beta particles and debris with decreasing radius, the negatively produced guiding center (main phase for MS) ExMF $F_{-M D E G}$ is given by

$$
E_{x M F_{-M D E G}}=B_{g}-\left(10^{8}\left(\gamma_{P S} B_{g x}^{2}+\frac{\gamma_{P S} n_{m} l q^{3} B_{g}^{3}}{m_{e}^{2} v_{c} c}\right)+10^{8}\left(\gamma_{P S} B_{g x}^{2}+\frac{\gamma_{P S} n_{m} l q^{3} B_{g}^{3}}{m_{p}^{2} v_{c} c}\right)\right) T
$$

Where, $E x M F_{-M D E}$ is the debris and beta particles negatively produced main phase, or the equivalent main phase for MS.

As Aurora was seen above Samoan, Fiji, and Tonga Islands [31], with the conjugate above Tongatapu [1], and synchrotrons radiation was observed at Wake Island, about $2500 \mathrm{~km}$ west of Johnston Island 10 minutes after the burst [3], with current interpretation described interaction of nuclear products (debris and electrons) with molecules to produce visible light, ultraviolet and infrared radiation [3], it is suggested that, with resulted small radius of gyration, cyclotron radiation is radiated as an aurora light in both southern and northern conjugates.

\section{Conclusion}

The paper is intended to show similarities in characteristics between the High-altitude Nuclear Detonation (HND) and the Magnetic Storm (MS), both mechanisms produced an intense External Magnetic Field Moment $\left(E x M F_{M}\right), H N D$ is also analyzed and related to the production of Interplanetary-External Magnetic Field Moment $\left(I-E x M F_{M}\right)$ in Magnetosheath during MS; a process intended to question a long time believe in solar magnetic field embedded in the solar wind, a theory which coined current human understanding of the magnetosphere interactions with solar wind, and many solar activities. It is also showed that, in the initial process leading to HND's $E x M F_{M}$ production an Electromagnetic Pulse (EMP) was generated, with characteristics similar to affects caused by Magnetic Storm (MS). The paper is seeking an alternative method capable of explaining different Astrophysical phenomena, and can help in solving many unsettled issues, like the energization of charged particles which is related to the production of External Magnetic Field (ExMF), both in which could help in resolving future energy crises.

\section{Acknowledgment}

I am grateful to the Reviewers of the Journal of Astrophysics \& Aerospace Technology for their valuable advices and Mustafa Mahmoud for editing the paper.

\section{References}

1. Glasstone Samuel (2006) EMP radiation from nuclear space bursts in 1962.

2. Francis N (1962) A Quick Look At The Technical Results Of Starfish Prime. Data Furnished by Kiley LA, Hoerlin H, Scott J, Gilbert FC, Strabala F. Unclassified Part of Mission Research Corporation report, USA

3. Herman H (1976) United States High-Altitude Test Experiences: A Review Emphasizing the Impact on the Environment. Los Alamos Scientific Laboratory, USA.

4. Johnston, Robert Wm (2009) High-altitude nuclear explosion Johnston archive, USA.

5. Wikipedia, High altitude Nuclear explosion.

6. YOSHIO K, TAKEI S (1963) Geomagnetic Disturbance accompanying the High Altitude Nuclear Detonation at Johnston Island, Japan.

7. Wikipedia, Nuclear Electromagnetic Pulse.

8. Berg RA, Holland DH, Futtermah WI (1967) A Starfish Happening. Ft. Belvoir Defense Technical Information Centre Publisher. Unites states.

9. Steven C, Crevier WF, Kilb RW (1979) MHDEMP Code Simulation of Starfish, Mission Research Corporation, USA.

10. Tatsuzo Obayashi (1963) Upper Atmosphere Disturbances Due to High Altitude Nuclear Explosions. Planet Space Sci 10: 47-63.

11. Wilson CR, Annexstad JO (1966) Study of Geomagnetic Micropulsations, Geophysical Institute of the University of Alasks, USA. 
Citation: Yousif ME (2014) Exploring the High-altitude Nuclear Detonation and Magnetic Storms. J Astrophys Aerospace Technol 2: 105. doi:10.4172/2329-6542.1000105

Page 8 of 8

12. Yousif ME (2012) Solar or Interplanetary External Magnetic Field.

13. Longmire CL (1986) Justification and Verification of High-Altitude EMP Theory, Part 1. Mission Research Corporation/Lawrence Livermore National Laboratory, USA.

14. Kamide Y (2007) Geomagnetic Storms. Solar-Terrestrial Environment Laboratory, Nagoya University, Japan.

15. Foster JS, Wood LL, Soper GK, Woodard JB, Graham WR, et al. (2008) Report of the Commission to assess the threat to the United States from Electromagnetic Pulse (EMP) Attack. United States.

16. Toffoletto F (1999) Geomagnetic Storms and Substorms, Rice University, USA.

17. Coleman PJ, Sonett CP, Davis L (1961) On the interplanetary magnetic storm: Pioneer V, J. Geophys Res 66: 7.

18. Ness NF (2001) Spacecraft studies of the interplanetary magnetic field. J Geophys Res 106: 15,803-15,817.

19. Fan CY, Meyer P, Simpson JA (1960) Rapid reduction of cosmic radiation intensity measured in interplanetary space. Phys Rev Letters 5: 269.

20. Yousif ME (2014) The Source of the Interplanetary Magnetic Field (IMF) Measured by Pioneer V.

21. Angelopoulos V, Sibeck D, Carlson CW, McFadden JP, Larson D, et al. (2009) First Results from the THEMIS Mission. Space Sci Rev 141: 453-476.

22. Gosling JT, Asbridge JR, Bame SJ, Strong IB (1967) Vela 2 measurements of the magnetopause and bow shock positions. J Geophys Res 72: 101-112.

23. Bieber JW, Stone EC (1982) Energetic Electrons in the Magnetosheath and Upstream of the Bow Shock. J Geophys Res 87: 85-94.

24. Watermann J, Wintoft P, Sanahuja B, Saiz E, Poedts S, et al. (2009) Models of Solar Wind Structures and Their Interaction with the Earth's Space Environment. Space Sci Rev 147: 233-270.

25. Lavraud B, Borovsky JE, Genot V, Schwartz SJ, Birn J, et al. (2009) Tracing solar wind plasma entry into the magnetosphere using ion-to-electron temperature ratio. Geophys Res Lett 36.

26. McDonald, Richard (2005) Planetary Magnetic Fields.

27. Syun-Ichi A, Chapman S (1961) The Ring Current, Geomagnetic Disturbance and the Van Allen Radiation Belts. J Geophys Res 66: 1321-1350.
28. Dyal P (1965) Operation Dominic. Fish Bowl Series. Debris Expansion Experiment. Air Force Weapons Laboratory, USAF, USA.

29. Kern JW (1967) Magnetosphere and Radiation Belt: Physics of Geomagnetic Phenomena. [2nd Ed] by Matsushita S, Campbell WH, Academic Press, N.Y, USA.

30. Yousif, ME (2003a) The Magnetic Interaction, Comprehensive Theory Articles Journal of Theoretics.

31. Emanuelson Jerry (2009) Operation Fishbowl-Futurescience.

32. McCorkle, Charles M, O'bryan CL, Prickett DI (1961) Preliminary Plan for Operation Fishbowl. Kirtland Air Force Base, New Mexico.

33. Russell CT, Wang YL, Raeder J, Tokar RL, Smith CW, et al. (2000) The interplanetary shock of September 24, 1998: Arrival at Earth. J Geophys Res 105: 25143-25154.

34. Yousif ME (2004) The Universal Energies, Journal of Theoretics.

35. Yousif ME (2003b) Elements of the Magnetic Lines Of Force, Journal of Theoretics.

36. Rastogi RG, Pathan BM, Rao DRK, Sastry TS, Sastri JH (2001) On latitudinal profile of Storm Sudden Commencement in H, Y and Z at Indian Geomagnetic Observatory chain. Earth Planets Space 53: 121-127.

37. Samuel G, Dolan PJ (1977) The Effects of Nuclear Weapons. [3rd Ed] U. S Department of Defense and U. S. Department of Energy,USA.

38. Wikipedia, Underground nuclear testing.

39. Longmire CL, Gilbert JL (1980) Theory of EMP Coupling in the Source Region Defense Nuclear Agency. report DNA 5687F, DTIC document reference ADA108751, California.

40. Seiler LW Jr (1975) A Calculational Model for High Altitude EMP, report ADA009208,Ohio,USA.

41. Longmire CL (1986) Justification and Verification of High-Altitude EMP Theory, Part 1, Lawrence Livermore National Laboratory, California.

\section{Wikipedia, Electric Field.}

43. Piddington JH (1967) A Hydromagnetic Model of Geomagnetic Storms and Auroras: Physics of Geomagnetic Phenomena. (2ndedn) By Matsushita S and Campbell WH. Academic Press, N. Y.

44. Le G, Russell CT, Takahashi K (2004) Morphology of the ring current derived from magnetic field observations. Ann Geophys 22: 1267-1295. 